\title{
ON MEROMORPHIC FUNCTIONS WITH FEW POLES AND WITH REGIONS FREE OF ZEROS
}

\author{
By Hideharu UEDA
}

1. Introduction. In this note we improve one of the results of Edrei and Fuchs [3]. We shall adopt the terminology, notations and conventions of [3]. We shall write, for instance, [3, Lemma 4] to denote Lemma 4 of [3].

The aim of this investigation is to prove the following

THEOREM. Suppose that $f(z)$ ( $\not \equiv$ const.) is a meromorphic function of lower order $\mu(<+\infty)$, and that $\delta(\infty, f)=1$. Let the $s$ B-regular paths

$$
\begin{aligned}
L_{l}: z & =t e^{2 \alpha_{l}(t)} \\
& \left(t \geqq t_{0}>0 ; l=1,2, \cdots, s ; \alpha_{1}(t)<\alpha_{2}(t)<\cdots<\alpha_{s}(t)<\alpha_{1}(t)+2 \pi=\alpha_{s+1}(t)\right)
\end{aligned}
$$

divide $|z| \geqq t_{0}$ into $s$ sectors, each of which has opening $\geqq c>0$.

Let $\delta(>0)$ be fixed and let $\bar{n}_{\delta}(r)$ denote the number of distinct zeros of $f(z)$ which lie in $t_{0} \leqq|z| \leqq r$ but outside the sectors $\mathcal{E}_{l}(\delta)(l=1,2, \cdots, s)$ defined by

$$
\alpha_{l}(t)-\delta \leqq \arg z \leqq \alpha_{l}(t)+\delta, \quad t_{0} \leqq|z|=t<+\infty .
$$

Assume that for every fixed $\delta(>0)$, we have

$$
\bar{n}_{\delta}(r)=o(T(r, f)) \quad(r \rightarrow \infty) .
$$

Denote by $p$ the number of deficient values of $f(z)$ other than 0 and $\infty$. Then

$$
p \leqq \min \left\{s-1,2 \mu,\{2 \mu(1-c / \pi)+1\}^{+}\right\} .
$$

This is an improvement of [3, Theorem 3].

\section{Proof of Theorem.}

2.1. Suppose that the function $f(z)$ satisfies the hypotheses of Theorem and it has $\tau_{1}, \tau_{2}, \cdots, \tau_{p}\left(\tau_{j} \neq 0, \tau_{j} \neq \infty, j=1,2, \cdots, p\right)$ among its deficient values.

The paths $L_{l}(l=1,2, \cdots, s)$ divide the $z$-plane into $s$ sectors $S_{l}$. Let $J_{l}(r)$ $\left(r \geqq t_{0}\right)$ be the set of arguments corresponding to the arc of $|z|=r$ in $S_{l}$. Since the $\tau$, are deficient, there is at least one index $l=l(j, r)$ such that for some

Received November 18, 1985 
fixed $\kappa>0$

$$
m\left(r, 1 /\left(f-\tau_{j}\right) ; J_{l}(r)\right)>\kappa T(r, f) \quad\left(r \geqq r_{0} \geqq t_{0}, l=l(j, r), j=1,2, \cdots, p\right) .
$$

With the equations (1.1) for the $L_{l}$, we shall denote by $S_{l}(\delta)(0 \leqq \delta<c / 16)$ the sector

$$
\alpha_{l}(t)+\delta<\arg z<\alpha_{l+1}(t)-\delta, \quad t_{0} \leqq|z|=t<+\infty ;
$$

by $J_{l}(r, \delta)$ the set of arguments of the arc $|z|=r$ in $S_{l}(\delta)$ and by $I_{l}(r, \delta)$ the complement of $J_{l}(r, \delta)_{i n} J_{l}(r, 0)=J_{l}(r)$.

Let $\left\{r_{m}\right\}_{1}^{\infty}$ be a sequence of Pólya peaks of order $\mu$ of $T(r, f)$ with associated sequences $\left\{r_{m}^{\prime}\right\}_{1}^{\infty},\left\{r_{m}^{\prime \prime}\right\}_{1}^{\infty}\left(r_{m}^{\prime}<r_{m}<r_{m}^{\prime \prime}, r_{1}^{\prime} \geqq r_{0}\right)$. (For the basic properties and existence of Pólya peaks the reader is referred to [2, p. 82].) Using [3, Lemma C], we have for $r_{m} \leqq r \leqq 2 r_{m}\left(m>m_{0}\right)$

$$
\begin{aligned}
m\left(r, 1 /\left(f-\tau_{j}\right) ; I_{l}(r, 2 \delta)\right) & \leqq 22 T\left(2 r, 1 /\left(f-\tau_{j}\right)\right) 4 \delta\left(1+\log ^{+}(1 / 4 \delta)\right) \\
& <89 T(2 r, f) \delta\left(1+\log ^{+}(1 / 4 \delta)\right) \\
& <90\left(2 r / r_{m}\right)^{\mu} T\left(r_{m}, f\right) \delta\left(1+\log ^{+}(1 / 4 \delta)\right) \\
& \leqq 90 \cdot 4^{\mu} T(r, f) \delta\left(1+\log ^{+}(1 / 4 \delta)\right) \\
& <(\kappa / 2) T(r, f),
\end{aligned}
$$

provided that $0<\delta<\delta_{1}=\delta_{1}(\kappa, \mu)$. Further, we may assume that $\delta_{1} \leqq \pi / 12 \mu$ s. Combining the above estimate with (2.1), we have

$$
\begin{aligned}
& m\left(r, 1 /\left(f-\tau_{j}\right) ; J_{l}(r, 2 \delta)\right)>(\kappa / 2) T(r, f) \\
& \quad\left(r_{m} \leqq r \leqq 2 r_{m}, m>m_{0}, 0<\delta<\delta_{1}, l=l(j, r), j=1,2 \cdots, p\right) .
\end{aligned}
$$

If $\mu<1 / 2$, the condition $\delta(\infty, f)=1$ implies that $p=0$ (See [4].). In what follows we assume that $\mu \geqq 1 / 2$. This gives

$$
\log r=o(T(r, f)) \quad(r \rightarrow \infty) .
$$

A basic fact of Nevanlinna's theory is that

$$
m\left(r, f^{\prime} /(f-a)\right)=o(T(r, f))
$$

as $r \rightarrow \infty$ outside an exceptional set $E$ which has finite measure. It is important to note that $E$ occurs in intervals where $T(r, f)$ grows very rapidly; in particular, $E$ does not depend on the value $a$, and consideration of the growth lemma from which it arises shows that it may be taken to be disjoint from the intervals $\left[r_{m}, \sigma r_{m}\right]\left(m>m_{0}\right)$, where $\sigma>1$ is fixed and $m_{0}=m_{0}(\boldsymbol{\sigma})$.

Combining (2.2), (2.3), [3, Lemma B] and the estimate

$$
\begin{array}{r}
m\left(r, f^{\prime} / f\right)+m\left(r, f^{\prime} /\left(f-\tau_{j}\right)\right)=o(T(r, f)) \\
\left(r_{m} \leqq r \leqq \sigma r_{m}, r \rightarrow \infty, j=1,2, \cdots, p\right),
\end{array}
$$


we obtain

$$
\begin{aligned}
& m\left(r, f / f^{\prime} ; J_{l}(r, 2 \delta)\right)>(\kappa / 3) T(r, f) \\
& \quad\left(r_{m} \leqq r \leqq 2 r_{m}, m>m_{0}, 0<\delta<\delta_{1}, l=l(\jmath, r), j=1,2, \cdots, p\right) .
\end{aligned}
$$

2.2. We choose now a constant $M(>0)$. For the proof of $p \leqq s-1$ we take $M=1$. For the proof of $p \leqq 2 \mu$ we shall obtain a contradiction if we assume $p>2 \mu$, and if we choose $M$ so large that

$$
e^{M}>16 A_{2}^{2}=U, \quad A_{2}=5 e^{4 \pi} / \pi,
$$

and

$$
-K / 2+6 \cdot 2^{\mu} A_{2} e^{-(p / 2-\mu) M}+4 A_{2} e^{-M / 2}<-K / 4,
$$

where the positive constant $K=K(\mu, c, s, B, \kappa)$ is defined in (2.33). If $p>2 \mu(1-$ $c / \pi)+1>0$, there is a number $c^{\prime} \in(c / 2, c)$ such that

$$
p>2 \mu\left(1-\left(3 c+c^{\prime}\right) / 4 \pi\right)+1 .
$$

In this case we choose $M$ so large that (2.6) and

$$
-K / 2+6 \cdot 2^{\mu} A_{2} \exp \left\{\left[-(p-1) / 2\left(1-\left(3 c+c^{\prime}\right) / 4 \pi\right)+\mu\right] M\right\}+4 A_{2} e^{-M / 2}<-K / 4
$$

hold. We shall seek a contradiction from (2.6), (2.8) and (2.9), and deduce $p \leqq$ $2 \mu(1-c / \pi)+1$.

2.3. By [3, Lemma 4]

$$
\left|f^{\prime}(z) / f(z)\right|<A\{T(2 r, f)\}^{A} \quad\left(|z|=r>r_{0}>4 \pi s B^{\prime} \delta_{1}, z \notin \mathcal{R}\right),
$$

where $\mathcal{R}$ is a set of discs with sum of radii less than 1 . Assume that $r_{m}^{\prime}<r$ $<r_{m}^{\prime \prime}$. It follows from (2.10) and the definition of Pólya peaks that

$$
\begin{aligned}
\left|f^{\prime}(z) / f(z)\right| & <A\left\{(1+o(1)) T\left(r_{m}, f\right)(2|z|)^{\mu} / r_{m}^{\mu}\right\}^{A} \\
& <2^{1+\mu A} A|z|^{\mu A}\left\{T\left(r_{m}, f\right) / r_{m}^{\mu}\right\}^{A} \quad\left(r_{m}^{\prime} \leqq|z| \leqq r_{m}^{\prime \prime} ; m>m_{0}, z \notin \mathscr{R}\right) .
\end{aligned}
$$

Hence we can find a positive integer $h$ such that

$$
\left|z^{-h} f^{\prime}(z) / f(z)\right|<\left\{T\left(r_{m}, f\right) / r_{m}^{\mu}\right\}^{A} \quad\left(r_{m}^{\prime} \leqq|z| \leqq r_{m}^{\prime \prime} ; m>m_{0}, z \notin \mathscr{R}\right) .
$$

It follows now from [3, Lemma 2] that there is some $\delta \in\left(\delta_{1} / 2, \delta_{1}\right)$ such that (2.11) holds on the boundaries of $S_{l}(\delta) \cap\left(\bigcup_{m=1}^{\infty}\left\{z ; r_{m}^{\prime} / 2<|z|<r_{m}^{\prime \prime} / 2\right\}\right)(l=1,2, \cdots, s)$. From now on we assume that $\delta$ has been chosen in this way and we shall make no further changes in the choice of $\delta$. It is also easily seen that there are two circles $|z|=R_{m}^{\prime}\left(r_{m} / 3 e^{M}<R_{m}^{\prime}<r_{m} / 2 e^{M}\right)$ and $|z|=R_{m}^{\prime \prime}\left(4 e^{M} r_{m}<R_{m}^{\prime \prime}<6 e^{M} r_{m}\right) \quad$ on which (2.11) holds.

Let 


$$
P_{m}(z)=\prod_{\nu=1}^{n_{m}}\left\{\left(z-a_{\nu}\right) / 2 R_{m}^{\prime \prime}\right\}
$$

be the product, taken over all the poles of $f^{\prime}(z) / f(z)$ which lie in $t_{0} \leqq|z| \leqq R_{m}^{\prime \prime}$ but outside the sectors $\mathcal{E}_{l}(\delta)(l=1,2, \cdots, s)$.

Now, we define a sequence of functions $\left\{g_{k}(z)\right\}_{m_{0}+1}^{\infty}$. Assume first that there is a subsequence $\left\{r_{m_{k}}\right\}_{k=1}^{\infty}$ of $\left\{r_{m}\right\}_{1}^{\infty}$ such that $T\left(r_{m_{k}}, f\right) / r_{m_{k}}^{\mu} \leqq 1$. In this case we simply write $\left\{r_{k}\right\}_{1}^{\infty},\left\{R_{k}^{\prime}\right\}_{1}^{\infty},\left\{R_{k}^{\prime \prime}\right\}_{1}^{\infty}$ and $\left\{P_{k}(z)\right\}_{1}^{\infty}$ instead of $\left\{r_{m_{k}}\right\}_{k=1}^{\infty}$, $\left\{R_{m_{k}}^{\prime}\right\}_{k=1}^{\infty},\left\{R_{m_{k}}^{\prime \prime}\right\}_{k=1}^{\infty}$ and $\left\{P_{m_{k}}(z)\right\}_{k=1}^{\infty}$, respectively, and define $g_{k}(z)(k \geqq 1)$ by

$$
g_{k}(z)=z^{-h} P_{k}(z) f^{\prime}(z) / f(z) .
$$

Assume next that $T\left(r_{m}, f\right) / r_{m}^{\mu}>1$ for $m>m_{0}$. In this case we define $g_{k}(z)$ $\left(k>m_{0}\right)$ by

$$
g_{k}(z)=\left\{r_{k}^{\mu} / T\left(r_{k}, f\right)\right\}^{A} z^{-h} P_{k}(z) f^{\prime}(z) / f(z) .
$$

The function $g_{k}(z)\left(k>m_{0}\right)$ is regular in the intersection of $|z| \leqq R_{k}^{\prime \prime}$ with every $S_{l}(\delta)(l=1,2, \cdots, s)$. In $|z| \leqq R_{k}^{\prime \prime}$

$$
\left|P_{k}(z)\right| \leqq 1 \text {. }
$$

By (2.11), (2.12) and the maximum modulus principle

$$
\left|g_{k}(z)\right|<1 \quad\left(z \in D_{k, l}\right),
$$

where $D_{k} l\left(k>m_{0}, l=1,2, \cdots, s\right)$ is defined by

$$
R_{k}^{\prime} \leqq r \leqq R_{k}^{\prime \prime}, \quad \alpha_{l}(r)+\delta \leqq \theta \leqq \alpha_{l+1}(r)-\delta .
$$

2.4. We first choose $c^{\prime \prime} \in(1,2)$ such that $\left(c^{\prime \prime}\right)^{\mu}<4 / 3$. Next, we select a positive number $b$ such that

$$
b<\min \left\{\left(c-c^{\prime}\right) / 144 \pi B e^{2 M+1},\left(c^{\prime \prime}-1\right) / 24 e^{M+1}\right\} .
$$

By a well-known lemma of $\mathrm{H}$. Cartan

$$
\left|P_{k}(z)\right| \geqq(b / 2)^{n_{k}}
$$

in $|z| \leqq R_{k}^{\prime \prime}$ but outside circles the sum of whose diameters is less than $4 e b R_{k}^{\prime \prime}$. It follows from [3, Lemma 2] and (2.14) that (2.15) holds on the curves $C_{k, l}$ $\left(k>m_{0}, l=1,2, \cdots, s\right)$ defined by

$$
C_{k, l}: z=z(t)=t e^{i\left(\alpha_{l}(t)+\gamma_{k}, l\right)} \quad\left(R_{k}^{\prime} \leqq t \leqq R_{k}^{\prime \prime}\right)
$$

with $c^{\prime} / 2<\gamma_{k, l}<c / 2$. Further, we deduce from (2.14) that (2.15) holds on a circle $|z|=R_{k}$ with $r_{k} \leqq R_{k} \leqq c^{\prime \prime} r_{k}\left(k>m_{0}\right)$. By (2.12) and (2.5)

$$
\begin{array}{r}
m\left(R_{k}, 1 / g_{k} ; J_{l}\left(R_{k}, 2 \delta\right)\right)>m\left(R_{k}, f / f^{\prime} ; J_{l}\left(R_{k}, 2 \delta\right)\right)>(\kappa / 3) T\left(R_{k}, f\right) \\
\left(k>m_{0}, l=l\left(j, R_{k}\right), \jmath=1,2, \cdots, p\right) .
\end{array}
$$


2.5. We note first, by repeating the argument following [3, (3.19)], that the image of $D_{k, l}\left(k>m_{0}, l=1,2, \cdots, s\right)$ by

$$
\zeta=\log z+\text { const. }
$$

contains a lens $\Lambda_{k, l}$ whose center line is formed by the vertical segment which is the image of $R_{k} e^{i \theta}\left(\theta \in J_{l}\left(R_{k}, \delta\right)\right)$ and whose boundary is formed by the two circular arcs through the endpoints of this segment making a sufficiently small constant angle $\beta$ with it. We choose $\beta=1 / 40 B$ and apply [3, Lemma 5] with $H(\zeta)=g_{k}(z), \quad \varepsilon=\delta \in\left(\delta_{1} / 2, \delta_{1}\right), \quad M^{*}=(\kappa / 3) T\left(R_{k}, f\right) \quad($ See $(2.16)),. \quad \alpha=\left\{\alpha_{l+1}\left(R_{k}\right)-\right.$ $\left.\alpha_{l}\left(R_{k}\right)\right\} / 2-\delta>3 c / 8$. This yields

$$
\log \left|g_{k}(z)\right|<-K_{1} T\left(R_{k}, f\right) \quad\left(z \in \mathscr{B}_{k}(l)\right),
$$

where $\mathscr{B}_{k}(l)$ is given by

$$
z=R_{k} e^{i \theta}, \quad \theta \in J_{l}\left(R_{k}, 2 \delta\right), \quad l=l\left(j, R_{k}\right), \quad j=1,2, \cdots, p,
$$

and where the constant $K_{1}$ may be chosen as

$$
K_{1}=\left(2 \kappa / 3 \pi^{3}\right)\left(\delta_{1} / 4 \pi\right)^{2 \pi / \beta} .
$$

Next, we apply [3, Lemma E], first to the part of $D_{k, l}$ in $|z| \geqq R_{k}$ then to the part of $D_{k, l}$ in $|z| \leqq R_{k}$. In both cases $\mathcal{B}_{k}(l)$ is the $\operatorname{arc}(2.18)$ and $\mathcal{L}$ is a portion of the curve $C_{k, l}$. It is easily verified, with the aid of [3, Lemma 1] that for any point $\zeta$ on $C_{k, l}$, with

$$
e^{-M} R_{k} \leqq|\zeta| \leqq e^{M} R_{k}
$$

we have

$$
\rho(\zeta)>|\zeta| / K_{2}(c, B) .
$$

From now on, we denote by $C_{k, l}^{\prime}$ the portion of $C_{k, l}$ which satisfies the condition (2.19). By (2.20) and the $B$-regularity of $C_{k, l}$

$$
\begin{array}{r}
\int_{w_{k, l}}^{z}|d \zeta| / \rho(\zeta) \leqq B K_{2}\left|\int_{R_{k}}^{|z|} d t / t\right|=B K_{2}\left|\log \left(|z| / R_{k}\right)\right| \\
\left(z \in C_{k, l}^{\prime}, w_{k, l}=R_{k} e^{\left.i \theta_{k, l} \in C_{k, l}^{\prime}\right) .}\right.
\end{array}
$$

Therefore, by (2.13), (2.17), [3, Lemma E] and the two-constant theorem

$$
\begin{array}{r}
\log \left|g_{k}(z)\right|<-\left(K_{1} / 2 \pi\right) \exp \left\{-4 B K_{2}\left|\log \left(|z| / R_{k}\right)\right|\right\} T\left(R_{k}, f\right) \\
\left(z \in C_{k, l}^{\prime}, k>m_{0}, l=l\left(j, R_{k}\right), j=1,2, \cdots, p\right) .
\end{array}
$$

2.6. We now deduce from (2.17) and (2.21) similar inequalities with $g_{k}$ replaced by $f^{\prime} / f$. Since $\delta(\infty, f)=1, n(r, \infty, f) \leqq(\log 2)^{-1} N(2 r, \infty, f)=o(T(2 r, f))$ $(r \rightarrow \infty)$. It follows from this, (1.2) and the definition of Pólya peaks that 


$$
\begin{aligned}
n_{k} & \leqq n\left(R_{k}^{\prime \prime}, \infty, f\right)+\bar{n}_{\delta}\left(R_{k}^{\prime \prime}\right)=o\left(T\left(2 R_{k}^{\prime \prime}, f\right) j\right. \\
& =o\left(T\left(12 e^{M} r_{k}, f\right)\right)=o\left(T\left(r_{k}, f\right)\right)=o\left(T\left(R_{k}, f\right)\right) \quad(k \rightarrow \infty) .
\end{aligned}
$$

By the definition of $g_{k}$

(2.23) $\log \left|f^{\prime}(z) / f(z)\right|=\log \left|g_{k}(z)\right|+h \log |z|-\log \left|P_{k}(z)\right|+A \log ^{+}\left\{T\left(r_{k}, f\right) / r_{k}^{\mu}\right\}$

Combining (2.23), (2.17), (2.18), (2.15), (2.3) and (2.22), we have

$$
\begin{aligned}
\log \left|f^{\prime}(z) / f(z)\right| & <-K_{1} T\left(R_{k}, f\right)+h \log R_{k}+n_{k} \log (2 / b)+A \log T\left(R_{k}, f\right) \\
& =-K_{1} T\left(R_{k}, f\right)+o\left(T\left(R_{k}, f\right)\right)<-\left(K_{1} / 2\right) T\left(R_{k}, f\right) \\
& \left(z \in \mathscr{B}_{k}(l), k>m_{0}, l=l\left(j, R_{k}\right), \jmath=1,2, \cdots p\right) .
\end{aligned}
$$

Similarly, using (2.21) instead of (2.17), we obtain

$$
\begin{array}{r}
\log \left|f^{\prime}(z) / f(z)\right|<-\left(K_{1} / 7\right) \exp \left\{-4 B K_{2}\left|\log \left(|z| / R_{k}\right)\right|\right\} T\left(R_{k}, f\right) \\
\left(z \in C_{k, l}^{\prime}, k>m_{0}, l=l\left(\jmath, R_{k}\right), j=1,2, \cdots, p\right) .
\end{array}
$$

By (2.2) with $r=R_{k}$, there is a point $z_{k, l} \in \mathscr{B}_{k}(l)\left(l=l\left(j, R_{k}\right), \jmath=1,2, \cdots, p\right)$ such that $\left|f\left(z_{k, l}\right)-\tau_{\jmath}\right|<\varepsilon$ for any assigned $\varepsilon>0$, provided that $k>m_{0}$. If $z$ is any other point of $\mathscr{B}_{k}(l)$, then by (2.24) and (2.3) $\left|\log f(z)-\log f\left(z_{k, l}\right)\right|<2 \pi R_{k} \times$ $\left.\exp \left\{-K_{1} / 2\right) T\left(R_{k}, f\right)\right\} \rightarrow 0(k \rightarrow \infty)$, and so for any assigned $\varepsilon \in(0,1 / 2)$

$$
\begin{aligned}
& \left|f(z)-\tau_{j}\right| \leqq\left|f(z)-f\left(z_{k, l}\right)\right|+\left|f\left(z_{k, l}\right)-\tau_{j}\right|<2 \varepsilon \\
& \quad\left(z \in \mathscr{B}_{k}(l), k>m_{0}, l=l\left(j, R_{k}\right), j=1,2, \cdots, p\right) .
\end{aligned}
$$

2.7. Since $r_{k} \leqq R_{k} \leqq c^{\prime \prime} r_{k}\left(k>m_{0}\right)$, we have for $r_{k}^{\prime} \leqq r \leqq r_{k}^{\prime \prime}$

$$
\begin{aligned}
T(r, f) / T\left(R_{k}, f\right) \leqq & T(r, f) / T\left(r_{k}, f\right) \leqq(1+o(1))\left(r / r_{k}\right)^{\mu} \\
& =(1+o(1))\left(R_{k} / r_{k}\right)^{\mu}\left(r / R_{k}\right)^{\mu} \leqq(1+o(1))\left(c^{\prime \prime}\right)^{\mu}\left(r / R_{k}\right)^{\mu} \\
& <(3 / 2)\left(r / R_{k}\right)^{\mu} \quad\left(k>m_{0}\right) .
\end{aligned}
$$

Let $\Lambda(r)=o(T(r, f))$ be a positive function tending to $\infty$ as $r \rightarrow \infty$. Then the following relation holds:

$$
\sigma_{k} \equiv \operatorname{meas}\left\{\theta ; \log \left|f\left(R_{k} e^{i \theta}\right)\right|>\Lambda\left(R_{k}\right)\right\}>\pi / 3 \mu \quad\left(k>m_{0}\right) .
$$

To prove this, we follow Baernstein's procedure in [1, pp. 430-434]. Let $\gamma=1 / 2 \mu$ and define

$$
v(z)=T^{*}\left(z^{r}\right) \quad\left(z=r e^{i \theta}, 0<r<\infty, 0 \leqq \theta \leqq \pi\right),
$$

where $T^{*}(z)$ is the Baernstein characteristic of $f$. Using (2.27) instead of [1, (4.8)], we obtain

$$
v\left(R_{k}^{1 / r} e^{i \theta}\right) \leqq(3 / 2) T\left(R_{k}, f\right)[\cos (\pi-\theta) \gamma \mu+o(1)] \quad(k \rightarrow \infty, 0<\theta<\pi),
$$


where the $o(1)$ term is independent of $\theta$. Clearly

$$
T^{*}\left(R_{k} e^{\imath \sigma_{k} / 2}\right) \leqq T\left(R_{k}, f\right)=m\left(R_{k}, f\right)+N\left(R_{k}, \infty, f\right) \leqq T^{*}\left(R_{k} e^{\imath \sigma_{k} / 2}\right)+\Lambda\left(R_{k}\right),
$$

and hence

$$
\lim _{k \rightarrow \infty} T^{*}\left(R_{k} e^{\imath \sigma_{k} / 2}\right) / T\left(R_{k}, f\right)=1 .
$$

Let $N=\left\{k ; \sigma_{k}<\pi \gamma\right\}$. If $N$ is a finite set then (2.28) holds, so we assume that $N$ is infinite. If $k \in N,\left(R_{k} e^{2 \sigma_{k} / 2}\right)^{1 / \gamma}=R_{k}^{1 / r} e^{2 \sigma_{k} / 2 r}$ belongs to the domain of $v$. In this case $T^{*}\left(R_{k} e^{2 \sigma_{k} / 2}\right)=v\left(R_{k}^{1 / \gamma} e^{2 \sigma_{k} / 2 \gamma}\right)(k \in N)$. Combining this, (2.29) and (2.30), we have

$$
\begin{array}{r}
T\left(R_{k}, f\right) \sim T^{*}\left(R_{k} e^{\imath \sigma_{k} / 2}\right) \leqq(3 / 2) T\left(R_{k}, f\right)\left[\cos \left(\pi-\sigma_{k} / 2 \gamma\right) \gamma \mu+o(1)\right] \\
(k \rightarrow \infty, k \in N),
\end{array}
$$

from which we deduce that $\sigma_{k}>\pi / 3 \mu\left(k \in N, k>m_{0}\right)$. If $k \notin N$, then $\sigma_{k} \geqq \pi \gamma=$ $\pi / 2 \mu>\pi / 3 \mu$. Thus we have reached (2.28).

2.8. By choosing $\varepsilon(>0)$ small enough, we see from (2.26) that the index $l=l\left(j, R_{k}\right)\left(k>m_{0}\right)$ cannot have the same value for different values of $j(=1,2, \cdots, p)$. This implies $p \leqq s$. Assume that $p=s$. Then from (2.28), (2.26) and (2.18) it follows that

$$
\pi / 3 \mu<\sigma_{k} \leqq 4 \delta s<4 \delta_{1} s \leqq \pi / 3 \mu \quad\left(k>m_{0}\right),
$$

which is impossible. This proves $p \leqq s-1$.

2.9. By integrating $f^{\prime} / f$ along $C_{k, l}\left(l=l\left(j, R_{k}\right)\right)$ from the point of intersection $w_{k, l}$ of $C_{k, l}^{\prime}$ with $\mathcal{B}_{k}(l)$ to the point $z$, we have, in view of (2.19) and (2.25)

$$
\begin{aligned}
\left|\log f(z)-\log f\left(w_{k, l}\right)\right|< & B\left(e^{M}-1\right) R_{k} \exp \left\{-\left(K_{1} / 7\right) e^{-4 B K_{2} M} T\left(R_{k}, f\right)\right\} \\
& \left(z \in C_{k, l}^{\prime}, k>m_{0}, l=l\left(j, R_{k}\right), j=1,2, \cdots, p\right) .
\end{aligned}
$$

Hence, by (2.26) and (2.3) we have, for any assigned $(\varepsilon>0)$

$$
\left|f(z)-\tau_{j}\right|<\varepsilon \quad\left(z \in C_{k, l}^{\prime}, k>m_{0}, l=l\left(\jmath, R_{k}\right), j=1,2, \cdots, p\right) .
$$

From this and (2.25) it follows that

$$
\begin{array}{r}
\log \left|f^{\prime}(z)\right|<-\left(K_{1} / 8\right) \exp \left\{-4 B K_{2}\left|\log \left(|z| / R_{k}\right)\right|\right\} T\left(R_{k}, f\right) \\
\left(z \in C_{k, l}^{\prime}, k>m_{0}, l=l\left(j, R_{k}\right), j=1,2, \cdots, p\right) .
\end{array}
$$

We have already seen that for any fixed $k\left(>m_{0}\right)$ the curves $C_{k, l}\left(l=l\left(j, R_{k}\right)\right.$, $j=1,2, \cdots, p)$ do not intersect, since they lie in different sectors $S_{l}(1 \leqq l \leqq s)$. Therefore they divide the annulus (2.19) into $p$ different domains.

Let $S_{k}^{*}$ be a typical one of these domains and let $t \Theta(t)$ be the length of the $\operatorname{arc}$ of $|z|=t$ which lies in $S_{k}^{*}$, and let 


$$
Q_{k}(z)=\prod_{\nu=1}^{n_{k}^{\prime}}\left\{\left(z-b_{\nu}\right) / 2 R_{k}^{\prime \prime}\right\}
$$

be the product, taken over all the poles of $f^{\prime}(z)$ which lie in $|z| \leqq R_{k}^{\prime \prime}$. By Cartan's lemma

$$
\left|Q_{k}(z)\right| \geqq(b / 2)^{n_{k}^{\prime}}
$$

in $|z| \leqq R_{k}^{\prime \prime}$ but outside circles the sum of whose diameters is less than $4 e b R_{k}^{\prime \prime}$. Without loss of generality, we may assume that (2.32) as well as (2.15) holds on the circle $|z|=R_{k}\left(k>m_{0}\right)$.

2.10. Let $A_{1}=e^{9 \pi}$ and let $A_{2}$ and $U\left(>A_{1}\right)$ be the quantities which appear in (2.6). Denote by $\Gamma_{1}(k, *)$ the part of the boundary of $S_{k}^{*}$ in $R_{k} / U<|z|<U R_{k}$, by $\Gamma_{2}(k, *)$ the boundary arc of $S_{k}^{*}$ on $|z|=e^{+M} R_{k}$, by $\Gamma_{3}(k, *)$ the boundary arc of $S_{k}^{*}$ on $|z|=e^{-M} R_{k}$ and by $\Gamma_{4}(k, *)$ the part of the boundary of $S_{k}^{*}$ which does not belong to $\Gamma_{1} \cup \Gamma_{2} \cup \Gamma_{3}$.

On $\Gamma_{1}$ and $\Gamma_{4}(2.31)$ holds, so that

$$
\begin{gathered}
\log \left|f^{\prime}(z) Q_{k}(z)\right|<-K T\left(R_{k}, f\right) \\
\left.\left(z \in \Gamma_{1}(k, *), K=\left(K_{1} / 8\right)\right) \exp \left\{-4 B K_{2} \log \left(16 A_{2}^{2}\right)\right\}=K(\mu, c, s, B, \kappa)\right), \\
\log \left|f^{\prime}(z) Q_{k}(z)\right|<0 \quad\left(z \in \Gamma_{4}(k, *)\right) .
\end{gathered}
$$

Since $f^{\prime}(z) Q_{k}(z)$ is regular in $|z| \leqq R_{k}^{\prime \prime}$, the Poisson-Jensen formula gives for $0<r<R_{k}^{\prime \prime} / 2$

Therefore by (2.4)

$$
\begin{aligned}
\log M\left(r, f^{\prime} Q_{k}\right) & \leqq 3 m\left(2 r, f^{\prime} Q_{k}\right) \leqq 3 m\left(2 r, f^{\prime}\right) \\
& \leqq 3 m\left(2 r, f^{\prime} / f\right)+3 m(2 r, f)
\end{aligned}
$$

$$
\begin{aligned}
\log \left|f^{\prime}(z) Q_{k}(z)\right| & \leqq \log M\left(e^{-M} R_{k}, f^{\prime} Q_{k}\right) \leqq \log M\left(R_{k} / 2, f^{\prime} Q_{k}\right) \\
& \leqq o\left(T\left(R_{k}, f\right)\right)+3 T\left(R_{k}, f\right)<4 T\left(R_{k}, f\right) \quad\left(z \in \Gamma_{3}(k, *)\right)
\end{aligned}
$$

and

$$
\begin{aligned}
\log \left|f^{\prime}(z) Q_{k}(z)\right| & \leqq o\left(T\left(2 e^{M} R_{k}, f\right)\right)+3 T\left(2 e^{M} R_{k}, f\right) \\
& <4 T\left(2 e^{M} R_{k}, f\right)<6\left(2 e^{M}\right)^{\mu} T\left(R_{k}, f\right) \quad\left(z \in \Gamma_{2}(k, *)\right) .
\end{aligned}
$$

Next, we denote by $\omega_{q}(z, k, *)$ the harmonic measure of $\Gamma_{q}(k, *)$ with respect to $S_{k}^{*}(q=1,2,3,4)$. Then by [3, Lemma 6],

$$
\begin{aligned}
\sum_{q=2}^{4} \omega_{q}\left(R_{k} e^{i \theta}, k, *\right) & <A_{2} \exp \left\{-\pi \int_{R_{k} / U}^{R_{k}} d t / t \Theta(t)\right\}+A_{2} \exp \left\{-\pi \int_{R_{k}}^{U R_{k}} d t / t \Theta(t)\right\} \\
& \leqq 2 A_{2} U^{-1 / 2}=1 / 2 \quad\left(R_{k} e^{i \theta} \in S_{k}^{*}\right)
\end{aligned}
$$

since $\Theta(t) \leqq 2 \pi$. Hence 


$$
\omega_{1}\left(R_{k} e^{i \theta}, k, *\right)>1 / 2 .
$$

Similarly,

$$
\omega_{3}\left(R_{k} e^{i \theta}, k, *\right)<A_{2} e^{-M / 2},
$$

and

$$
\omega_{2}\left(R_{k} e^{\imath \theta}, k, *\right)<A_{2} \exp \left\{-\pi \int_{R_{k}}^{e^{M} R_{k}} d t / t \Theta(t)\right\} .
$$

We show that for at least one of the sectors, say for $S_{k}^{* *}$,

$$
\pi \int_{R_{k}}^{e^{M} R_{k}} d t / t \Theta(t) \geqq p M / 2
$$

and, say for $S_{k}^{* * *}$,

$$
\pi \int_{R_{k}}^{e^{M} R_{k}} d t / t \Theta(t) \geqq(p-1) M / 2\left(1-\left(3 c+c^{\prime}\right) / 4 \pi\right) .
$$

By Schwarz's inequality and the fact that $\sum_{j=1}^{p} \Theta_{\jmath}(t)=2 \pi$ (where the index $\jmath$ refers to the $p$ different sectors $S_{k}^{*}$ )

$$
p^{2}=\left\{\sum_{j=1}^{p}\left(\Theta_{\jmath}(t)\right)^{1 / 2}\left(\Theta_{j}(t)\right)^{-1 / 2}\right\}^{2} \leqq 2 \pi \sum_{j=1}^{p} 1 / \Theta_{j}(t) .
$$

Hence

$$
p^{2} M / 2=\left(p^{2} / 2\right) \int_{R_{k}}^{e^{M_{R_{k}}}} d t / t \leqq \sum_{j=1}^{p} \pi \int_{R_{k}}^{e^{M} R_{k}} d t / t \Theta_{j}(t) .
$$

This proves (2.40). In the same way, using the facts that $p \leqq s-1$ and $c^{\prime} / 2<$ $\gamma_{k, l}<c / 2\left(k>m_{0}, l=1,2, \cdots, s\right)$, we have

$$
(p-1)^{2} \leqq\left(2 \pi-\left(3 c+c^{\prime}\right) / 2\right) \sum_{j=1}^{p-1} 1 / \Theta_{j}(t) .
$$

Hence

$$
(p-1)^{2} M / 2=\left((p-1)^{2} / 2\right) \int_{R_{k}}^{e^{M} R_{k}} d t / t \leqq \sum_{j=1}^{p-1}\left(\pi-\left(3 c+c^{\prime}\right) / 4\right) \int_{R_{k}}^{e^{M} R_{k}} d t / t \Theta_{j}(t) .
$$

This proves (2.41). Combining (2.39) with (2.40) or (2.41), we have

$$
\omega_{2}\left(R_{k} e^{i \theta}, k, * *\right)<A_{2} e^{-p M / 2} \quad\left(R_{k} e^{i \theta} \in S_{k}^{* *}\right),
$$

and

$$
\begin{array}{r}
\omega_{2}\left(R_{k} e^{i \theta}, k, * * *\right)<A_{2} \exp \left\{-(p-1) M / 2\left(1-\left(3 c+c^{\prime}\right) / 4 \pi\right)\right\} \\
\left(R_{k} e^{i \theta} \in S_{k}^{* * *}\right) .
\end{array}
$$

2.11. Now, a bounded function, harmonic in $S_{k}^{*}$, with the following boundary values 


$$
\begin{array}{ll}
-K T\left(R_{k}, f\right) \text { on } \Gamma_{1}(k, *), & 6 \cdot 2^{\mu} e^{\mu M} T\left(R_{k}, f\right) \text { on } \Gamma_{2}(k, *), \\
4 T\left(R_{k}, f\right) \text { on } \Gamma_{3}(k, *), \quad 0 & \text { on } \Gamma_{4}(k, *)
\end{array}
$$

dominates the subharmonic function $\log \left|f^{\prime}(z) Q_{k}(z)\right|$ at each point of $S_{k}^{*}$. This follows from (2.33)-(2.36). Hence, in particular,

$$
\begin{aligned}
\log \left|f^{\prime}\left(R_{k} e^{i \theta}\right) Q_{k}\left(R_{k} e^{i \theta}\right)\right| & \\
< & -\omega_{1}\left(R_{k} e^{i \theta}, k, *\right) K T\left(R_{k}, f\right)+\omega_{2}\left(R_{k} e^{i \theta}, k, *\right) 6 \cdot 2^{\mu} e^{\mu M} T\left(R_{k}, f\right) \\
& +\omega_{3}\left(R_{k} e^{i \theta}, k, *\right) 4 T\left(R_{k}, f\right) \quad\left(R_{k} e^{i \theta} \in S_{k}^{*}, k>m_{0}\right) .
\end{aligned}
$$

From (2.44), (2.37), (2.42), (2.38), (2.7) and (2.32) we deduce that

(2.45) $\log \left|f^{\prime}\left(R_{k} e^{i \theta}\right)\right|$

$$
\begin{array}{r}
<\left\{-K / 2+6 \cdot 2^{\mu} A_{2} e^{-(p / 2-\mu) M}+4 A_{2} e^{-M / 2}\right\} T\left(R_{k}, f\right)-\log \left|Q_{k}\left(R_{k} e^{i \theta}\right)\right| \\
<-(K / 4) T\left(R_{k}, f\right)+n_{k}^{\prime} \log (2 / b)<-(K / 5) T\left(R_{k}, f\right) \\
\quad\left(k>m_{0}, R_{k} e^{i \theta} \in S_{k}^{* *}\right),
\end{array}
$$

where we used the estimate

$$
\begin{aligned}
n_{k}^{\prime}=n\left(R_{k}^{\prime \prime}, \infty, f^{\prime}\right) & \leqq 2 n\left(R_{k}^{\prime \prime}, \infty, f\right)=o\left(T\left(2 R_{k}^{\prime \prime}, f\right)\right)=o\left(T\left(12 e^{M} r_{k}, f\right)\right) \\
& =o\left(T\left(r_{k}, f\right)\right)=o\left(T\left(R_{k}, f\right)\right) \quad(k \rightarrow \infty) .
\end{aligned}
$$

Similarly, from (2.44), (2.37), (2.43), (2.38), (2.9) and (2.32) it follows that

$$
\begin{aligned}
& \log \mid f^{\prime}\left(R_{k} e^{i \theta}\right) \mid<\left\{-(K / 2)+6 \cdot 2^{\mu} A_{2} \times\right. \\
&\left.\exp \left[\left\{-(p-1) / 2\left(1-\left(3 c+c^{\prime}\right) / 4 \pi\right)+\mu\right\} M\right] 4 A_{2} e^{-M / 2}\right\} T\left(R_{k}, f\right) \\
&-\log \left|Q_{k}\left(R_{k} e^{i \theta}\right)\right|<-(K / 4) T\left(R_{k}, f\right)+n_{k}^{\prime} \log (2 / b)<-(K / 5) T\left(R_{k}, f\right) \\
& \quad\left(k>m_{0}, R_{k} e^{i \theta} \in S_{k}^{* * *}\right) .
\end{aligned}
$$

2.12. Let $\zeta_{k, 1}$ and $\zeta_{k, 2}$ be the endpoints of the $\operatorname{arc}$ of $|z|=R_{k}$ in $S_{k}^{* *}\left(S_{k}^{* * *}\right)$. Then we easily see from (2.26) that

$$
\left|f\left(\zeta_{k, 1}\right)-f\left(\zeta_{k, 2}\right)\right|>\min _{\substack{\imath \neq \jmath \\ \imath, j=1,2 \cdots, p}}\left|\tau_{\imath}-\tau_{j}\right| / 2 \quad\left(k>m_{0}\right) .
$$

On the other hand, by integrating $(2.45)((2.46))$

$$
\left|f\left(\zeta_{k, 1}\right)-f\left(\zeta_{k, 2}\right)\right| \leqq 2 \pi R_{k} \exp \left\{-(K / 5) T\left(R_{k}, f\right)\right\}
$$

and, in view of (2.3), the right - hand side of this inequality tends to 0 as $k \rightarrow \infty$. This contradicton proves $p \leqq 2 \mu(p \leqq 2 \mu(1-c / \pi)+1)$. This completes the proof of Theorem. 


\section{REFERENCES}

[1] Baernstein II, A., Proof of Edrei's spread conjecture, Proc. London Math. Soc. 26 (1973), 418-434.

[2] EdREI, A., Sums of deficiencies of meromorphic functions, J. Analyse Math. 14 (1965) , 79-107.

[3] EdRei, A. AND Fuchs, W. H.J., On meromorphic functions with regions free of poles and zeros, Acta Math. 108 (1962), 113-145.

[4] OstrovskiI, I. V., Deficiencies of meromorphic functions of order less than one, Dokl. Akad. Nauk SSSR 150 (1963), 32-35.

Department of Mathematics

DAIDO InSTITUTE OF TECHNOLOGY

DaIDo-Cho, Minami-KU, Nagoya

JAPAN 